\title{
Actitud y percepción estudiantil con el uso de la tecnología en la universidad
}

\section{Student's Attitude and Perception with the Use of Technology in the University}

\author{
Francisco Orgaz* (D) Universidad Tecnológica de Santiago, Santiago de los Caballeros, \\ República Dominicana \\ ORCID: https://orcid.org/0000-0002-4240-8640
}
Salvador Moral iD Universidad Tecnológica de Santiago, Santiago de los Caballeros, República Dominicana
ORCID: https://orcid.org/0000-0003-0853-7032
Cándida M. Domínguez Universidad Tecnológica de Santiago, Santiago de los Caballeros, República Dominicana ORCID: https://orcid.org/0000-0003-0778-5579

Recibido 23-07-18 Revisado 14-08-18 Aprobado 11-10-18 En línea 16-10-18

\begin{tabular}{|c|c|}
\hline *Correspondencia & Citar como: \\
\hline Email: franorgaz@utesa.edu & $\begin{array}{l}\text { Orgaz, F., Moral, S., \& Domínguez, C.M. } \\
\text { (2018). Actitud y percepción estudiantil con el } \\
\text { uso de la tecnología en la universidad. Propósitos } \\
\text { y Representaciones, 6(2), 253-299. Doi: http:// } \\
\text { dx.doi.org/10.20511/pyr2018.v6n2.230 }\end{array}$ \\
\hline
\end{tabular}

(C) Universidad San Ignacio de Loyola, Vicerrectorado de Investigación, 2018

(cc) BY-NC-ND Este artículo se distribuye bajo licencia CC BY-NC-ND 4.0 Internacional (http://creativecommons.org/licenses/by-nc-nd/4.0/). 


\section{Resumen}

Este estudio tiene como finalidad conocer la relación existente entre las variables actitud hacia las redes sociales, actitud hacia la tecnología, percepción de la tecnología y uso de la tecnología de los estudiantes universitarios. La metodología empleada ha consistido en la aplicación de un cuestionario, el cual ha sido aplicado a estudiantes de la Universidad Tecnológica de Santiago (UTESA) de República Dominicana. Los datos han sido analizados a través de los programas estadísticos SPSS y SmartPLS (técnica Partial Least Square -PLS-). Entre los principales resultados, se ha podido comprobar que la actitud hacia la tecnología por parte de los estudiantes influye en la percepción que tiene sobre la tecnología, y que la actitud de los estudiantes ante las redes sociales influye positivamente en el uso de la tecnología.

Palabras clave: Tecnología, herramientas, enseñanza superior, actitudes de los estudiantes, República Dominicana. 


\section{Summary}

This study aims to know the relationship between the variables attitude toward social networks, attitude toward technology, perception about technology and use of technology by university students. The methodology used has been a questionnaire, which has been applied to students of the Technological University of Santiago (UTESA) of the Dominican Republic. The data has been analyzed through the statistical programs SPSS and SmartPLS (Partial Least Square technique -PLS-). Among the main results, it has been found that students 'attitude toward technology influences their perception about technology, and that students' attitude toward social networks has a positive influence on the use of technology.

Keywords: Technology, tools, higher education, student's attitudes, Dominican Republic. 


\section{Introducción}

El término tecnología hace referencia a cualquier tipo de aplicación, incluido el hardware, software, ordenadores, bases de datos, Internet y correo electrónico (Tubaishat, Al-Rawajfah, Habiballah \& Akhu-Zaheya, 2016), es decir, este concepto es amplio y se utiliza para referirse a aplicaciones de hardware y software empleadas para almacenar, crear, intercambiar y usar información (Nkosi, Asah \& Pillay, 2011). Desde la introducción de las primeras computadoras personales en la década de 1960, se han producido avances significativos en la tecnología que conducen a la adopción rápida de dispositivos personales y móviles (Law, Thome, Lindeman, Jackson \& Lidor, 2018). A medida que el uso de dispositivos tecnológicos móviles ha proliferado, también lo hace el concepto de que tales dispositivos pueden ser útiles en el proceso de enseñanza y aprendizaje (Eppard, Nasser \& Reddy, 2016). El uso de la tecnología móvil en la educación brinda a los educadores la oportunidad de re-imaginar la enseñanza y el aprendizaje (Heflin, Shewmaker \& Nguyen, 2017). Esto crea un modelo de aprendizaje más flexible que brinda a los miembros de la facultad y estudiantes acceso a múltiples fuentes de información y un cambio de una estructura de aprendizaje basada en el concepto de una comunidad de aprendices (Hamm, Saltsman, Jones, Baldridge \& Perkins, 2013).

El uso de tecnologías móviles en la educación y el aprendizaje se ha investigado en los últimos años (Briz-Ponce, Juanes-Mendez \& GarcíaPenalvo, 2014; Huang, Lin \& Chuang, 2007), demostrándose que el aprendizaje móvil es beneficioso para aumentar la independencia, el compromiso y la comunicación de los estudiantes (Dunn, Richardson, Oprescu $\&$ McDonald, 2013). Así, el uso de dispositivos móviles en el aprendizaje mejora el compromiso al proporcionar acceso inmediato a la información, proporcionando un aprendizaje práctico mejorado (Cheng, Yang, Chang \& Kuo, 2016), aunque los dispositivos móviles son más propicios para el aprendizaje cuando la instrucción ha sido cuidadosamente diseñada para hacer un uso óptimo de la tecnología (Heflin, Shewmaker \& Nguyen, 2017). 
En este sentido, la Organización de las Nacionales Unidas (UNESCO, 2009) indicó que la tecnología tiene un gran potencial en la calidad del aprendizaje y mejora los buenos resultados de los estudiantes. Los dispositivos móviles y las aplicaciones educativas no deben complicar el proceso de aprendizaje, sino facilitar dicho aprendizaje en los estudiantes (Jeng, Wu, Huang, Tan \& Yang, 2010). Con este fin, los docentes están utilizando aplicaciones móviles en el aula, debido a que permiten respuestas de los estudiantes a las preguntas del instructor en función del contenido del curso (Heflin, Lin \& Chuang, 2017), demostrándose que esto mejora las percepciones de los estudiantes sobre el aprendizaje (Denker, 2013). El uso de estas aplicaciones brinda a los docentes la oportunidad de utilizar la tecnología móvil para promover un aprendizaje significativo (Heflin, Lin \& Chuang, 2017). La integración de dispositivos móviles en el aprendizaje de los estudiantes ha indicado que el efecto general del uso de dispositivos móviles parece ser mejor para el aprendizaje que el uso de computadoras de escritorio o no usar dispositivos en absoluto (Sung, Change \& Liu 2016).

Los estudiantes de hoy en día son nativos digitales (Palfrey \& Gasser, 2011), lo que aumenta la importancia de que los docentes desarrollen estrategias de aprendizaje cómodas (Denker, Manning, Heuett \& Summers, 2018), por ejemplo, a través de la tecnología. Esta forma de participación amplía el aula de la universidad en espacios virtuales (Denker, Hermann $\&$ Willits, 2015), donde los estudiantes están conectados a través de las tecnologías de la información y la comunicación (Denker, Manning, Huett \& Summers, 2018), influyendo esto de forma positiva en el proceso de enseñanza-aprendizaje (Pando, 2018). La tecnología ayuda, además, a que los estudiantes participen en el aula desde otros espacios (Denker, 2013). En este sentido, Finn \& Ledbetter (2013) encontraron que los estudiantes universitarios desean que sus docentes les permitan usar la tecnología en el aula. Ledbetter (2009) concluyó que las actitudes de comunicación en línea por parte de los estudiantes como una forma de orientación cognitiva y afectiva puede fomentar o inhibir la tendencia de un individuo a comunicarse 
en línea. Guo, Li \& Stevens (2012) ofrecieron un modelo de actitudes que dan forma al uso de la tecnología, aunque esos modelos no explican las relaciones entre las actitudes y el uso de la tecnología. En este sentido, a medida que más clases continúan agregando elementos tecnológicos, como el uso de las redes sociales (Tyma, 2011), se hace necesario examinar las actitudes de los estudiantes frente a la tecnología y el aprendizaje, sobre todo, con el aumento de la popularidad de los medios sociales (DomínguezVergara \& Ybañez-Carranza, 2016).

Los factores que podrían influir en el comportamiento de los estudiantes para usar tecnologías en el aprendizaje se han considerado un elemento atractivo para el desarrollo de muchas investigaciones (Briz-Ponce, Pereira, Carvalho, Juanes-Méndez \& García-Peñalvo, 2017). Por ejemplo, Hong, Thong \& Tam (2006) hacen una comparación entre tres modelos para comprender el comportamiento de uso de Internet móvil. Sánchez y Hueros (2010) realizaron un análisis de las plataformas virtuales de enseñanza para el aprendizaje a distancia y el uso y aceptación de la tecnología. Por su parte, los hallazgos publicados por Chen (2011) muestran que la compatibilidad educativa y la expectativa son determinantes importantes de la aceptación del e-learning. Espuny, González, Lleixá \& Gisbert (2011) encontraron relación favorable entre las actitudes de los estudiantes y el uso de las redes sociales. Arteaga, Duarte \& García (2013) investigaron los factores que determinan la aceptación del sistema de aprendizaje online entre los estudiantes. Lee \& Lehto (2013) realizaron una investigación basada en un modelo de aceptación de la tecnología y la intención conductual de utilizar nuevas tecnologías. Thakre \& Thakre (2015) explicaron cuáles son los principales usos de los teléfonos inteligentes por parte de los estudiantes y concluyeron que la comunicación, el aprendizaje y el entretenimiento son los más populares. Por otro lado, Sezer (2016) reveló que los factores de género y el éxito académico afectan significativamente a las actitudes de los estudiantes hacia el aprendizaje y la tecnología. Por su parte, Briz-Ponce, Pereira, Carvalho, Juanes-Méndez \& García-Peñalvo (2017) encontraron una fuerte actitud de los estudiantes universitarios hacia el uso y recomendación de la tecnología móvil. 
Cheung \& Vogel (2013) concluyeron que la actitud positiva del usuario conducirá a una mayor intención de utilizar la tecnología para el aprendizaje. Sujeet \& Jyoti (2013) postularon que una mayor intención conductual puede verse influenciada por la actitud y la facilidad de uso percibida entre los estudiantes. Por su parte, Rupak, Greg, Jei \& Ben (2014) encontraron que la tecnología tiene una relación positiva y significativa entre la utilidad percibida y la facilidad de uso percibida, y ambos elementos tienen un efecto positivo en la intención conductual. En definitiva, la actitud juega un papel importante en la persuasión de la intención de los estudiantes de utilizar el aprendizaje en línea (Hussein, 2017), ya que la actitud es un componente vital en el uso de la tecnología (Altawallbeh, Soon, Thiam \& Alshourah, 2015). El uso de herramientas de la Web 2.0, como la Wikipedia, también ha puesto de manifiesto que mejora significativamente algunas competencias básicas de los estudiantes, como son la interacción y la comunicación, la comprensión lectora, la escritura o la capacidad de investigación (Soler, Pavlovic \& Font, 2018).

De esta manera, el estudio plantea la problemática de que las variables actitud hacia la tecnología y las redes sociales no han sido analizadas, en profundidad, con la percepción que tienen los estudiante frente a la tecnología, lo que puede generar inadecuados comportamientos de la web 2.0 en la universidad y, por consiguiente, un desaprovechamiento de los recursos tecnológicos para mejorar el proceso de enseñanza-aprendizaje. Así, resulta pertinente profundizar en la relación de estas variables, con la misión de generar implicaciones que fomenten un uso adecuado de la tecnología y los recursos tecnológicos en la universidad. Por esta razón, la literatura científica mencionada en los párrafos anteriores apoya las siguientes hipótesis: H1, la actitud hacia la tecnología influye positivamente en la percepción de la tecnología; H2, la actitud hacia las redes sociales influye positivamente en la percepción de la tecnología; H3, la actitud hacia las redes sociales influye positivamente en el uso de la tecnología; y H4, la percepción de la tecnología influye positivamente en el uso de la tecnología. Para conocer si estas 
hipótesis son soportadas, se ha realizado un trabajo de campo cuantitativo con los estudiantes de la Universidad Tecnológica de Santiago (UTESA, en adelante), en República Dominicana. De esta manera, el objetivo de esta investigación es conocer la relación existente entre las variables actitud hacia las redes sociales, actitud hacia la tecnología, percepción de la tecnología y uso de la tecnología de los estudiantes universitarios.

\section{Método}

\section{Diseño, estructura y participantes.}

Los participantes de este estudio han sido los estudiantes de UTESA, localizada en República Dominicana. Estos estudiantes pertenecen a la Sede-Central situada en la localidad de Santiago de los Caballeros, la cual cuenta con 21.147 estudiantes de grado (UTESA, 2017). El estudio fue llevado a cabo entre los meses de enero y junio de 2017 en las aulas de la universidad, empleando un enfoque cuantitativo a través de un muestreo aleatorio simple, recogiendo un total de 693 cuestionarios, aunque finalmente solamente resultaron ser válidos 660 cuestionarios. Con todo ello, se obtuvo un error muestral del 3.8\%, empleándose un nivel de confianza del 95\%. El cuestionario se aplicó mediante la técnica de muestreo aleatorio simple, donde todo el universo tiene las mismas opciones de ser seleccionada para rellenar el cuestionario (Casas, Repullo \& Donado, 2003).

El cuestionario estaba debidamente estructurado, llevándose a cabo un proceso de validación consistente en tres pasos: en el primero de estos, los ítems que han sido incluidos en el cuestionario han sido seleccionados de estudios previos (Barczyk \& Duncan, 2013; Cao, Ajjan \& Hong, 2013; Eid \& Al-Jabri, 2016; Pintado, 2015; Spiegel \& Rodríguez, 2016); posteriormente, dos expertos en la materia revisaron el cuestionario con el objetivo de corroborar que el procedimiento del diseño del instrumento se realizó de forma correcta; en el tercer y último paso se aplicó un pretest de 20 encuestas a estudiantes de UTESA, buscando detectar posibles desviaciones o errores 
en la interpretación de los cuestionarios, ya que estos fueron facilitados en dos idiomas diferentes (castellano y francés), con la finalidad de llegar a la mayor cantidad de estudiantes, ya que la gran mayoría son de procedencia dominicana (habla castellana) o haitiana (habla francesa).

La estructura del cuestionario se divide en dos partes claramente diferenciadas. En la primera parte, es abordado el uso de la tecnología y las redes sociales por parte de los estudiantes en la universidad, llevándose a cabo esta valoración mediante una escala de Likert de cinco (5) puntos (donde 1 hace referencia a "muy en desacuerdo"; 3 es "punto de indiferencia"; y 5 significa "muy de acuerdo"). En la segunda parte, se abordan preguntas relativas al perfil sociodemográfico. El número total de ítems fue de 25.

\section{Procedimiento de análisis de datos.}

El proceso de tabulación y análisis previo estadístico consistió en la comprobación de la fiabilidad de los ítems a través del alfa de Cronbach. Este procedimiento fue llevado a cabo mediante el paquete estadístico IBM SPSS v.24. En este sentido, en el proceso de depuración de ítems mediante alfa de Cronbach dio como resultado la eliminación de 7 ítems del cuestionario, debido a que su correlación elemento-total corregido era inferior a 0,3 (Norussis, 1993). Tras esto, el alfa de Cronbach válido para los 18 ítems restantes fue de 0.781 , siendo válida la escala por ser superior a 0,7 (Nunnally \& Bernstein, 1994).

Tras el análisis previo basado en el uso del programa estadístico IBM SPSS v.24, se procedió al uso de otro programa estadístico de Ecuaciones Estructurales basados en la varianza, a través del uso de los mínimos cuadrados parciales (en inglés Partial Least Squares, PLS) mediante el programa SmartPLS v.3.2.6. La elección de esta técnica de análisis PLS se realizó porque este programa permite comprobar si los conceptos iniciales teóricos se han medido de forma correcta mediante las diferentes variables observadas incluidas en el modelo, analizándose la validez y la fiabilidad de 
estos. Así, a la hora de llevar a cabo un análisis mediante mínimos cuadrados parciales (PLS), se plantean dos etapas muy claramente diferenciadas (Barclay, Higgins \& Thompson, 1995). En una primera etapa, se realiza la evaluación de la fiabilidad y validez del modelo de medida y, en una segunda etapa, se desarrolla la evaluación del modelo estructural.

\section{Resultados}

Para obtener una mejor compresión de los resultados, estos se han dividido en tres partes. Primeramente, se presenta los resultados descriptivos del perfil sociodemográfico de la muestra. En segundo lugar, se muestra la primera etapa del modelo PLS: evaluación de la fiabilidad y validez del modelo de medida. Y, en tercer lugar, se muestra la evaluación del modelo estructural.

\section{Resultados descriptivos previos.}

En la tabla 1 se muestran los resultados relativos al perfil sociodemográfico de la muestra de estudiantes universitarios. Cabe destacar un mayor número de estudiantes de género femenino (66.2\%) que masculino (33.8\%), siendo el tramo de edad más representativo el situado entre los 18 y 25 años (88.2\%). El 90.2\% tiene la nacionalidad dominicana. La estadía en la universidad más representada es aquella comprendida entre 1 y 3 años (50.5\%), siendo el grado universitario más cursado por los encuestados el de Medicina (39.7\%), seguido de Odontología (9.6\%), Contaduría Pública (9.4\%) e Ingeniería Civil (9.0\%), entre otras. Se comprobó que el teléfono móvil o celular se conforma como el dispositivo más empleado en el ámbito universitario, representando por un $75.6 \%$ de las respuestas, seguido del ordenador portátil o laptop (15.0\%). 


\section{Tabla 1.}

Perfil sociodemográfico de la muestra.

\begin{tabular}{|c|c|c|c|}
\hline Variable & $\%$ & Variable & $\%$ \\
\hline $\begin{array}{l}\text { Género }(N=642) \\
\text { Hombre } \\
\text { Mujer }\end{array}$ & $\begin{array}{l}33.8 \% \\
66.2 \%\end{array}$ & $\begin{array}{l}\text { Años en la universidad }(N=638) \\
\text { Menos de } 1 \text { año } \\
1-3 \text { años } \\
\text { Más de } 3 \text { años }\end{array}$ & $\begin{array}{l}22.9 \% \\
50.5 \% \\
26.6 \%\end{array}$ \\
\hline $\begin{array}{l}\text { Edad }(N=462) \\
18-25 \text { años } \\
26-34 \text { años } \\
\text { 35-44 años } \\
45-54 \text { años } \\
\text { 55-64 años } \\
\text { Más de } 65 \text { años }\end{array}$ & $\begin{array}{c}88.2 \% \\
10.6 \% \\
1.1 \% \\
0.0 \% \\
0.0 \% \\
0.1 \%\end{array}$ & $\begin{array}{l}\text { Carrera }(N=647) \\
\text { Medicina } \\
\text { Odontología } \\
\text { Contaduría Pública } \\
\text { Ingeniería Civil } \\
\text { Psicología } \\
\text { Bioanálisis } \\
\text { Resto carreras }\end{array}$ & $\begin{array}{c}39.7 \% \\
9.6 \% \\
9.4 \% \\
9.0 \% \\
4.8 \% \\
3.1 \% \\
24.4 \%\end{array}$ \\
\hline $\begin{array}{l}\text { País de procedencia } \\
(N=633) \\
\text { República Dominicana } \\
\text { República de Haití } \\
\text { México } \\
\text { Estados Unidos } \\
\text { Venezuela }\end{array}$ & $\begin{array}{c}90.2 \% \\
9.0 \% \\
0.3 \% \\
0.3 \% \\
0.2 \%\end{array}$ & $\begin{array}{l}\text { Dispositivo más usado }(N=419) \\
\text { Celular } \\
\text { Computadora } \\
\text { Laptop } \\
\text { Tablet } \\
\text { Reloj inteligente }\end{array}$ & $\begin{array}{c}75.6 \% \\
6.0 \% \\
15.0 \% \\
1.7 \% \\
1.7 \%\end{array}$ \\
\hline
\end{tabular}

\section{Evaluación de la fiabilidad y validez del modelo de medida.}

En la tabla 2 se presentan los compuestos modo A (Percepción Tecnológica -PT- y Uso de la Tecnología -UT-) y modo B (Actitud hacia la Tecnología -AT- y Actitud hacia las Redes Sociales -ARS-). En lo relativo a los compuestos modo A se ha procedido a su validez y fiabilidad a través de las cargas factoriales, eliminándose las cargas que presentasen valores inferiores a 0,7 (Cepeda \& Roldán, 2004). De esta manera, fueron eliminados tres ítems previamente. También ha de tenerse en cuenta la consistencia interna, medida a través de la fiabilidad compuesta y el alfa de Cronbach (Werts, Linn y Jöreskog, 1974), aunque la medida más fiable y de mejor aplicación para la consistencia interna es la fiabilidad compuesta, ya que no se encuentra influida por el número de ítems que pueda tener la escala (Fornell \& Larcker, 1981). Para que exista fiabilidad compuesta, los compuestos han de presentar valores de este índice superiores a 0,7 (Henseler, Hubona \& Ray, 2016). La Validez Convergente también ha de ser tenida en cuenta en este proceso de validez del modelo de medida y, para ello, los valores de la Varianza Extraída 
Media (AVE, en inglés) han de ser superiores a 0.5 (Fornell \& Larcker, 1981). Posteriormente, para el cálculo de la Validez Discriminante, se acude a las cargas cruzadas, debiendo ser, las cargas entre ítems del mismo compuesto, superiores a las cargas respecto a los otros compuestos. Por último, la ratio Heterotrait-Monotrait (HT-MT) es una medida más exigente (Henseler, Hubona \& Ray 2016), que tiene la finalidad de determinar la existencia de validez discriminante. Para ello, ha de presentar valores inferiores a 0.85 (Kline, 2011) o a 0,90 (Teo, Srivastava \& Jiang, 2008).

Resulta importante destacar que los índices anteriores son solo de aplicación para compuestos modo A. En el caso de los compuestos modo $\mathrm{B}$, se analizan a través de sus pesos, indicándonos la importancia relativa que cada ítem tiene en la formación de su compuesto. También ha de tenerse en cuenta la posible existencia de multicolinealidad entre estos ítems pertenecientes a los compuestos modo B. La determinación de existencia de esta multicolinealidad es medida a través del Test del Factor de Inflación de la Varianza (FIV en inglés), indicando la existencia de este problema valores superiores a 5 (Hair, Sarstedt, Hopkins \& Kuppelwieser, 2014) o a 3.3 (Roberts \& Thatcher, 2009). En este sentido, en las tablas 2 y 3 se presentan los resultados del proceso de validez y fiabilidad del modelo de medida, donde no se observan valores anómalos en ninguno de los índices y ratios calculados, encontrándose todos por encima del valor mínimo exigido, por lo que la validez y fiabilidad del modelo de medida es adecuada y correcta. 
Tabla 2.

Cargas, pesos, FIV, Consistencia Interna y Validez Convergente.

\begin{tabular}{|c|c|c|c|c|c|}
\hline & $\begin{array}{l}\text { Fiabilidad } \\
\text { Compuesta }\end{array}$ & AVE & Cargas factoriales & Pesos & FIV \\
\hline $\begin{array}{l}\text { Percepción tecnológica } \\
\text { (PT) } \\
\text { PT1 } \\
\text { PT4 }\end{array}$ & 0.824 & 0.702 & $\begin{array}{l}0.907 \\
0.762\end{array}$ & & \\
\hline $\begin{array}{l}\text { Uso de la tecnología (UT) } \\
\text { UT2 } \\
\text { UT8 }\end{array}$ & 0.851 & 0.742 & $\begin{array}{l}0.789 \\
0.928\end{array}$ & & \\
\hline $\begin{array}{l}\text { Actitud hacia la tecnología } \\
\text { (AT) } \\
\text { AT6 } \\
\text { AT7 } \\
\text { AT15 } \\
\text { AT16 }\end{array}$ & - & - & & $\begin{array}{l}0.605 \\
0.353 \\
0.167 \\
0.160\end{array}$ & $\begin{array}{l}1.625 \\
1.277 \\
1.322 \\
1.295\end{array}$ \\
\hline $\begin{array}{l}\text { Actitud hacia las Redes Sociales } \\
\text { (ARS) } \\
\text { ARS17 } \\
\text { ARS18 } \\
\text { ARS19 } \\
\text { ARS20 }\end{array}$ & s & - & & $\begin{array}{l}0.158 \\
0.113 \\
0.040 \\
0.823\end{array}$ & $\begin{array}{l}1.331 \\
1,465 \\
2.299 \\
2.452\end{array}$ \\
\hline
\end{tabular}

Tabla 3.

Validez discriminante.

\begin{tabular}{ccccc}
\hline Cargas cruzadas & PT & UT & AT & ARS \\
\hline PT1 & $\mathbf{0 . 9 0 7}$ & 0.555 & 0.540 & 0.544 \\
PT4 & $\mathbf{0 . 7 6 2}$ & 0.372 & 0.312 & 0.368 \\
UT2 & 0.368 & $\mathbf{0 . 7 8 9}$ & 0389 & 0.381 \\
UT8 & 0.588 & $\mathbf{0 . 9 2 8}$ & 0.558 & 0.614 \\
AT6 & 0.481 & 0.481 & $\mathbf{0 . 9 0 9}$ & 0.552 \\
AT7 & 0.379 & 0.445 & $\mathbf{0 . 7 1 6}$ & 0.419 \\
AT15 & 0.322 & 0.359 & $\mathbf{0 . 6 0 9}$ & 0.389 \\
AT16 & 0.316 & 0.350 & $\mathbf{0 . 5 9 7}$ & 0.455 \\
ARS17 & 0.330 & 0.330 & 0.458 & $\mathbf{0 . 5 6 9}$ \\
ARS18 & 0.365 & 0.372 & 0.319 & $\mathbf{0 . 6 3 5}$ \\
ARS19 & 0.461 & 0.430 & 0.548 & $\mathbf{0 . 7 6 7}$ \\
ARS20 & 0.543 & 0.594 & 0.595 & $\mathbf{0 . 9 8 1}$ \\
\multicolumn{5}{c}{ Ratio Heterotrait-Monotrait: 0.831} \\
\hline
\end{tabular}




\section{Evaluación del modelo estructural.}

Para llevar a cabo una estimación exacta del modelo de medida se emplea la técnica del bootstrapping (Roldán \& Sánchez-Franco, 2012), obteniéndose un valor $t$ y la probabilidad límite asociada para cada una de las hipótesis a contrastar. En la tabla 4 se presenta el contraste de las hipótesis planteadas, donde se observa que la actitud hacia la tecnología por parte de los estudiantes influye en la percepción que tiene sobre la tecnología (H2). También, se ha podido soportar que la actitud de los estudiantes antes las redes sociales influyen positivamente en el uso de la tecnología (H3). En el caso de la existencia de influencia positiva de la actitud hacia la percepción tecnológica (H1) y entre la percepción tecnológica y el uso de la tecnología (H4), estas no han sido soportada.

\section{Tabla 4.}

Contraste de hipótesis.

\begin{tabular}{lcccc}
\hline & $\begin{array}{c}\text { Coeficiente } \\
\text { Path }\end{array}$ & Valor $t$ & $\begin{array}{c}\mathrm{p} . \\
\text { límite }\end{array}$ & ¿Soportada? \\
\hline $\begin{array}{l}\text { H1: Actitud hacia la tecnología } \rightarrow \text { Percepción } \\
\text { tecnológica }\end{array}$ & $0.298^{\mathrm{NS}}$ & 2.265 & .012 & No \\
$\begin{array}{l}\text { H2: Actitud hacia las redes sociales } \rightarrow \text { Percepción } \\
\text { tecnológica }\end{array}$ & $0.374^{*}$ & 2.488 & .006 & Sí \\
$\begin{array}{l}\text { H3: Actitud hacia las redes sociales } \rightarrow \text { Uso de la } \\
\text { tecnología }\end{array}$ & $0.412^{*}$ & 2.368 & .009 & Sí \\
H4: Percepción tecnológica $\rightarrow$ Uso de la tecnología & $0.338^{\mathrm{NS}}$ & 2.180 & .015 & No \\
\hline$* * * \mathrm{p}<.001, * * \mathrm{p}<.005, * \mathrm{p}<0,01 .\left(\mathrm{t}_{(659)}\right.$, una cola). $\mathrm{t}(.001 ; 659)=3.090 ; \mathrm{t}(.005 ; 659)=2.576 ; \mathrm{t}(0.01 ; 659)$ \\
$=2.326 . \mathrm{NS}=$ no significativo
\end{tabular}

Los resultados obtenidos ponen de manifiesto el impacto de las variables exógenas sobre las endógenas. Así, la actitud hacia la tecnología explica un $15.794 \%$ de la variable percepción tecnológica. Las actitudes hacia las redes sociales explican un $20.862 \%$ de la variabilidad de la percepción tecnológica y un $24.761 \%$ de la varianza de la variable uso de la tecnología. Finalmente, la percepción tecnológica explica un 19,198\% de la varianza del uso de la tecnología. Por otro lado, la información sobre el poder predictivo del modelo viene dado por el coeficiente de determinación o R² (Hair, Sarstedt, 
Hopkins \& Kuppelwieser, 2014), si bien, el test de Stone-Geiser $\left(\mathrm{Q}^{2}\right)$ brinda una mayor relevancia predictiva (Stone, 1974). Un valor de $Q^{2}$ de StoneGeisser superior a 0 indica relevancia predictiva para el compuesto, mientras que un valor inferior a 0 indica ausencia de relevancia predictiva de dicho compuesto (Henseler, Ringle \& Sinkovics, 2009). Para esta investigación, los constructos endógenos presentan valores de $\mathrm{Q}^{2}$ superiores a $0\left(\mathrm{Q}^{2}\right.$ Percepción tecnológica $=0.135 ; \mathrm{Q}^{2}$ Uso de la tecnología $\left.=.018\right)$. De esta manera, el modelo final queda presentado en la figura 1 .

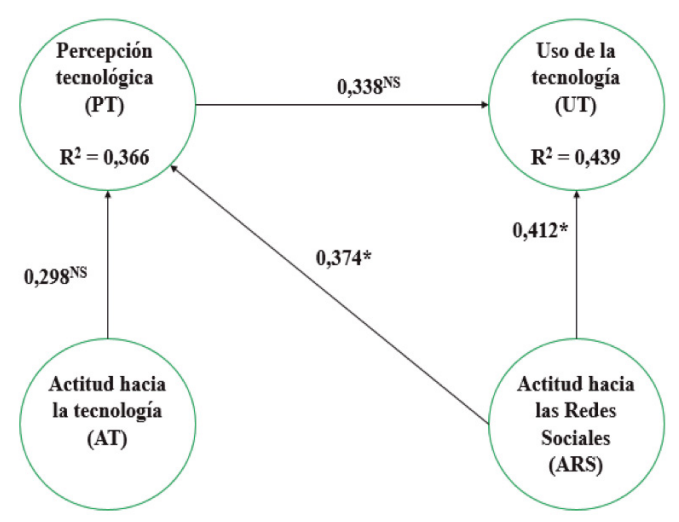

Figura 1. Modelo estructural final

\section{Discusión}

El principal hallazgo de este estudio es que el modelo de estudio planteado en esta investigación soporta que la actitud hacia la tecnología por parte de los estudiantes influye en la percepción que tiene sobre la tecnología. También, se puede concluir que la actitud de los estudiantes ante las redes sociales influye positivamente en el uso de la tecnología. Estos resultados confirman un modelo donde se explican las relaciones entre las actitudes y el uso de la tecnología, algo que anteriormente no había sido confirmado (Guo, Li \& Stevens, 2012). Estos resultados, además, indican la existencia de relación entre la actitud y el uso de la tecnología, hipótesis soportada en otros estudios (Cheung \& Vogel, 2013; Briz-Ponce, Pereira, Carvalho, 
Juanes-Méndez \& García-Peñalvo 2017; Rupak, Greg, Jei, \& Ben, 2014). Por el contrario, no se ha podido comprobar que la actitud hacia la tecnología influya positivamente en la percepción tecnológica de los estudiantes, ni la percepción tecnológica influya en el uso de la tecnología por parte de los alumnos. Como implicaciones para la gestión, estos resultados pueden servir a la universidad para formular estrategias que fomenten las actitudes positivas hacia la utilización de la Web 2.0 en el proceso de enseñanza-aprendizaje entre su comunidad de estudiantes y docentes, puesto que se ha comprobado que existe una relación positiva entre ambos elementos. También, puede servir para desarrollar políticas de buenas prácticas para la utilización de las plataformas webs y, sobre todo, para que la comunidad académica conozca los beneficios de la utilización de la tecnología y las redes sociales en el proceso de enseñanza-aprendizaje.

Entre las principales limitaciones de este estudio, podemos citar que la temporalidad del trabajo de campo ha sido corta, debido a que se han recolectado datos solamente en los primeros seis meses del año $2017 \mathrm{y}$, por tanto, hubiera sido más interesante aplicar el cuestionario a muestras de alumnos en un periodo más largo de tiempo. También, el estudio está enfocado solamente desde el punto de vista de los estudiantes, sin conocer la percepción de la utilización de la Web 2.0 por parte de otros actores académicos, como docentes, investigadores o directivos universitarios. Por otro lado, es posible que la extensión del cuestionario haya podido generar alguna incidencia en la calidad de las respuestas de los visitantes. En este sentido, y con el objetivo de salvar este inconveniente, el proceso de depuración ha sido muy exhaustivo, eliminando todos los cuestionarios que presentaran dudas en la veracidad de sus respuestas. Como futuras líneas de investigación, resultaría interesante indagar en que aspectos o labores de la universidad utilizan los estudiantes la Web 2.0. También, sería prudente conocer otros aspectos relacionados con los comportamientos de los estudiantes en la Web 2.0 y el uso en la universidad, como elementos relacionados con el estrés académico en el proceso enseñanza-aprendizaje y la influencia o beneficios de la Web 
2.0 en los aspectos que ayudan a combatir ese estrés. Por último, sería recomendable aplicar el cuestionario en otras universidades para conocer sí las relaciones del modelo son soportadas o no y, por tanto, obtener una mayor información sobre la relación entre las variables del estudio.

\section{Referencias}

Altawallbeh, M., Soon, F., Thiam, W., \& Alshourah, S. (2015). Mediating Role of Attitude, Subjective Norm and Perceived Behavioural Control in the Relationships between Their Respective Salient Beliefs and Behavioural Intention to Adopt ELearning among Instructors in Jordanian Universities. Journal of Education and Practice, 6(11), 152-159.

Arteaga, R., Duarte, A., \& García, M. (2013). E-learning and the University of Huelva: a study of WebCT and the technological acceptance model. Campus-wide In-formation Systems, 30(2), 135-160. Doi: https://doi. org/10.1108/10650741311306318

Barclay, D., Higgins, C., \& Thompson, R. (1995). The partial least squares (PLS) approach to casual modeling: personal computer adoption and use as a 356 illustration. Technology Studies, Special Issue on Research Methodology, 2(2), 285-309.

Barczyk, C., \& Duncan, D. (2013). Facebook in higher education courses: An analysis of students' attitudes, community of practice, and classroom community. International Business and Management, 6(1), 1-11. Doi: http://dx.doi.org/10.3968/j.ibm.1923842820130601.1165

Briz-Ponce, L., Pereira, A., Carvalho, L., Juanes-Méndez, J. A., \& GarcíaPeñalvo, F. J. (2017). Learning with mobile technologies-Students' behavior. Computers in Human Behavior, 72, 612-620. Doi: https://doi. org/10.1016/j.chb.2016.05.027

Briz-Ponce, L., Juanes-Mendez, J. A., \& García-Penalvo, F. J. (2014). A systematic review of using mobile devices in medical education. En J. Sierra-Rodriguez, J. Dodero-Beardo, y D. Burgos (Eds.), Proceedings of 2014 international sympo- sium on computers in education (SIIE) (pp. 205-210). Logroño: Institute of Electrical and Electronics Engineers Inc. Doi: http://dx.doi.org/10.1109/SIIE.2014.7017731 
Cao, Y., Ajjan, H., \& Hong, P. (2013). Using social media applications for educational outcomes in college teaching: A structural equation analysis. British Journal of Educational Technology, 44(4), 581-593. Doi: http:// dx.doi.org/10.1111/bjet.12066

Casas Anguita, J., Repullo Labrador, J.R. \& Donado Campos, J. (2003). La encuesta como técnica de investigación. Elaboración de cuestionarios y tratamiento estadístico de los datos (I). Atención Primaria, 31(8), 527538. Doi: https://doi.org/10.1016/S0212-6567(03)70728-8

Cepeda Carrión, G., \& Roldán Salgueiro, J.L. (2004). Aplicando la técnica PLS en la Administracción de Empresas. En XIV Congreso ACEDE: Conocimiento y Competitividad. Murcia: ACEDE.

Chen, J.L. (2011). The effects of education compatibility and technological expectancy on e-learning acceptance. Computers and Education, 57(2), 1501-1511. Doi: http://dx.doi.org/10.1016/j.compedu.2011.02.009

Cheng, P., Yang, Y. C., Chang, S. G., \& Kuo, F. R. (2016). 5E Mobile inquiry learning approach for enhancing learning motivation and scientific inquiry ability of university students. IEEE Transactions on Education, 59(2), 147-153. Doi: https://doi.org/10.1109/TE.2015.2467352

Cheung, R., \& Vogel, D. (2013). Predicting user acceptance of collaborative technologies: An extension of the technology acceptance model for e-learning. Computers \& Education, 63, 160-175. Doi: https://doi. org/10.1016/j.compedu.2012.12.003

Denker, K. J. (2013). Student response systems and facilitating the large lecture basic communication course: Assessing engagement and learning. Communication Teacher, 27, 50-69. Doi: https://doi.org/10.10 80/17404622.2012.730622

Denker, K. J., Hermann, A. F., \& Willits, M. D. D. (2015). Technology as engagement: How we learn and teach while polymediating the classroom. En A. Tyma, A. Herbig, y A. F. Hermann (Eds.), Beyond new media: Discourse and critique in a polymediated age (pp. 143-161). Lanham: Lexington Press.

Denker, K. J., Manning, J., Heuett, K. B., \& Summers, M. E. (2018). Twitter in the classroom: Modeling online communication attitudes and student motivations to connect. Computers in Human Behavior, 79, 1-8. Doi: https://doi.org/10.1016/j.chb.2017.09.037 
Domínguez-Vergara, J. A., \& Ybañez-Carranza, J. (2016). Adicción a las redes sociales y habilidades sociales en estudiantes de una institución educativa privada. Propositos y Representaciones, 4(2), 181-230. Doi: http://dx.doi.org/10.20511/pyr2016.v4n2.122

Dunn, P., Richardson, A., Oprescu, F., \& McDonald, C. (2013). Mobilephone-based classroom response systems: Students' perceptions of engagement and learning in a large undergraduate course. International Journal of Mathematical Education in Science and Technology, 44(8), 1160-1174. Doi: https://doi.org/10.1080/0020739X.2012.756548

Eid, M., \& Al-Jabri, I. (2016). Social networking, knowledge sharing, and student learning: The case of university students. Computers \& Education, 99, 14-27. Doi: https://doi.org/10.1016/j. compedu.2016.04.007

Eppard, J., Nasser, O., \& Reddy, P. (2016). The next generation of technology: Mobile apps in the English language classroom. International Journal of Emerging Technologies in Learning, 11(4), 21-27. Doi: http://dx.doi. org/10.3991/ijet.v11i04.5293

Espuny Vidal, C., González Martínez, J., Fortuño, M. L., \& Gisbert Cervera, M. (2011). Actitudes y expectativas del uso educativo de las redes sociales en los alumnos universitarios. RUSC. Universities and Knowledge Society Journal, 8(1), 171-185.

Finn, A. N., \& Ledbetter, A. M. (2013). Teacher power mediates the effects of technology policies on teacher credibility. Communication Education, 62, 26-47. Doi: https://doi.org/10.1080/03634523.2012.725132

Fornell, C., \& Larcker, D.F. (1981). Evaluating structural equations models with unobservable variables and measurement error. Journal of Marketing Research, 18, 39-50. Doi: https://doi.org/10.2307/3151312

Guo, Z., Li, Y., \& Stevens, K. J. (2012). Analyzing students' technology use motivations: An interpretive structural modeling approach. Communications of the Association for Information Systems, 30(1), 14-23.

Hair, J.F., Sarstedt, M., Hopkins, L., \& Kuppelwieser, V. (2014). Partial Least Squares structural equation modeling (PLS-SEM). An emerging tool in business research. European Business Review, 26(2), 106-121. Doi: https://doi.org/10.1108/EBR-10-2013-0128 
Hamm, S., Saltsman, G., Jones, B., Baldridge, S., \& Perkins, S. (2013). A mobile pedagogy approach for transforming learners and faculty. En Zane Berge, y Lin Muilenburg (Eds.), Handbook of mobile education. New York: Routledge.

Heflin, H., Shewmaker, J., \& Nguyen, J. (2017). Impact of mobile technology on student attitudes, engagement, and learning. Computers \& Education, 107, 91-99. Doi: https://doi.org/10.1016/j. compedu.2017.01.006

Henseler, J., Ringle, C.M., \& Sinkovics, R.R. (2009). The use of partial least squares path modeling in international maketing. Advances in International Marketing, 20, 277-319. Doi: https://doi.org/10.1108/ S1474-7979(2009)0000020014

Henseler, J., Hubona, G., \& Ray, P.A. (2016). Using PLS path modeling in new technology research: updated guidelines. IMDS, 116(1), 2-20. Doi: https://doi.org/10.1108/IMDS-09-2015-0382

Hong, S., Thong, J. Y. L., \& Tam, K. Y. (2006). Understanding continued information technology usage behavior: a comparison of three models in the context of mobile internet. Decision Support Systems, 42(3), 18191834. Doi: http://dx.doi.org/10.1016/j.dss.2006.03.009

Huang, J.H., Lin, Y.R., \& Chuang, S.T. (2007). Elucidating user behavior from mobile learning: a perspective of the extended technology acceptance model. The Electronic Library, 25(5), 585-598. Doi: https:// doi.org/10.1108/02640470710829569

Hussein, Z. (2017). Leading to Intention: The Role of Attitude in Relation to Technology Acceptance Model in E-Learning. Procedia Computer Science, 105, 159-164. Doi: https://doi.org/10.1016/j.procs.2017.01.196

Jeng, Y. L., Wu, T. T., Huang, Y. M., Tan, Q., \& Yang, S. J. (2010). The add-on impact of mobile applications in learning strategies: A review study. Educational Technology \& Society, 13(3), 3-11.

Kline, R. B. (2011). Principles and practice of structural equation modelling. New York: Guildford Press.

Law, J. K., Thome, P. A., Lindeman, B., Jackson, D. C., \& Lidor, A. O. (2018). Student use and perceptions of mobile technology in clinical clerkships-Guidance for curriculum design. The American 
Journal of Surgery, 215(1), 196-199. Doi: https://doi.org/10.1016/j. amjsurg.2017.01.038

Ledbetter, A. M. (2009). Measuring online communication attitudes: Instrument development and validation. Communication Monographs, 76, 463-468. Doi: https://doi.org/10.1080/03637750903300262

Lee, D. Y., \& Lehto, M. R. (2013). User acceptance of YouTube for procedural learning: an extension of the technology acceptance model. Computers \& Education, 61, 193-208. Doi: http://dx.doi.org/10.1016/j. compedu.2012.10.001

Nkosi, Z. Z., Asah, F., \& Pillay, P. (2011). Post basic nursing students' access to and attitudes toward the use of information technology in practice: a descriptive analysis. Journal of Nursing Management, 19(7), 876-882. Doi: http://dx.doi.org/10.1111/j.1365-2834.2011.01303.x

Norussis, M.J. (1993). SPSS. Statistical Data Analysis. New York: SPSS Inc.

Nunnally, J.C., \& Bernstein, I. (1994). Psychometric theory. New York: McGraw-Hill.

Palfrey, J. G., \& Gasser, U. (2011). Born digital: Understanding the first generation of digital natives. New York: Basic.

Pando, V. F. (2018). Tendencias didácticas de la educación virtual: Un enfoque interpretativo. Propósitos y Representaciones, 6(1), 463-505. Doi: http://dx.doi.org/10.20511/pyr2018.v6n1.167

Roberts, N., \& Thatcher, J. (2009). Conceptualizing and testing formative constructs: Tutorial and annotated example. ACM SIGMIS Database, 40(3), 3-39. Doi: https://doi.org/10.1145/1592401.1592405

Roldán, J.L., \& Sánchez-Franco, M.J. (2012). Variance-based structural equation modeling: guidelines for using partial least squares in information systems research. En Mora, M., Gelman, O., Steenkamp, A., Raisinghani, M.S. (Eds.), Research Methodologies, Innovations and Philosophies in Software Systems Engineering and Information Systems (pp. 193-221). Hershey: IGI Group. Doi: https://doi.org/10.4018/978-14666-0179-6.ch010 
Pintado Blanco, T. (2015). Las aplicaciones móviles interactivas en el aula: sus efectos en el aprendizaje y en el nivel de satisfacción del alumnado. Madrid: Universidad Complutense de Madrid.

Rupak, R., Greg, R., Jei, Y. \& Ben, J. (2014). Technology acceptance model (TAM) and social media usage: an empirical study on Facebook. Journal of Enterprise Information Management, 27(1), 6-30. Doi: https://doi. org/10.1108/JEIM-04-2012-0011

Sánchez, R. A., \& Hueros, A. D. (2010). Motivational factors that influence the acceptance of Moodle using TAM. Computers in Human Behavior, 26(6), 1632-1640. Doi: http://dx.doi.org/10.1016/j.chb.2010.06.011

Sezer, B. (2016). Faculty of medicine students' attitudes towards electronic learning and their opinion for an example of distance learning application. Computers in Human Behavior, 55, 932-939. Doi: http:// dx.doi.org/10.1016/j.chb.2015.10.018

Soler,J.,Pavlovic,D.,\&Font,P.F.(2018).Wikipediaenla Universidad:cambios en la percepción de valor con la creación de contenidos. Comunicar: Revista científica iberoamericana de comunicación y educación, (54), 39-48. Doi: https://doi.org/10.3916/C54-2018-04

Spiegel, A., \& Rodríguez, G. (2016). Students at university have mobile technologies. Do they do m-learning?. Procedia-Social and Behavioral Sciences, 217, 846-850. Doi: https://doi.org/10.1016/j. sbspro.2016.02.006

Stone, M. (1974). Cross-validatory choice and assessment of statistical predictions. Journal of the Royal Statistical Society, 36(2), 111-147.

Sujeet, K.S. \& Jyoti, K.C. (2013). Technology acceptance model for the use of learning through websites among students in Oman. International Arab Journal of E-Technology, 3(1),44-49, 2013.

Sung, Y.-T., Change, K.-E., \& Liu, T.-C. (2016). The effects of integrating mobile devices with teaching and learning on students' learning performance: A meta-analysis and research synthesis. Computer and Education, 94, 252-275. Doi: https://doi.org/10.1016/j. compedu.2015.11.008

Teo, T.S.H., Srivastava, S.C., \& Jiang, L. (2008). Trust and electronic government success: An empirical study. Journal of Management 
Information Systems, 25(3), 99-132. Doi: https://oi.org/10.2753/ MIS0742-1222250303

Thakre, S., \& Thakre, S. (2015). Perception of medical students for utility of mobile technology use in medical education. International Journal of Medicine and Public Health, 5(4), 305-311. Doi: http://dx.doi. org/10.4103/2230-8598.165959

Tubaishat, A., Al-Rawajfah, O. M., Habiballah, L., \& Akhu-Zaheya, L. M. (2016). Exploring changes in nursing students' attitudes towards the use of technology: A four-wave longitudinal panel study. Nurse education today, 38, 101-106. Doi: http://dx.doi.org/10.1016/j.nedt.2015.12.006

Tyma, A. (2011). Connecting with what is out there! Using Twitter in the large lecture. Communication Teacher, 25, 175-181. Doi: https://doi.org /10.1080/17404622.2011.579911

UNESCO. (2009). La nueva dinámica de la educación superior y la investigació para el cambio social y el desarrollo. In Conferencia Mundial sobre la Educación Superior. París: UNESCO.

UTESA (2017). Infográfico de UTESA 2017. Santiago de los Caballeros: Universidad Tecnológica de Santiago.

Werts, C.E., Linn, R.L. \& Jöreskog, K.G. (1974). Interclass reliability estimates: testing structural assumptions. Educational and Psychological Measurement, 34, 25-33. Doi: https://doi. org/10.1177/001316447403400104 